\title{
Consulting the Digital Natives
}

\section{ADAM STAPLETON}

UNIVERSITY OF WESTERN SYDNEY

\section{Sonia Livingstone}

Children and the Internet: Great Expectations, Challenging Realities

Polity, Cambridge and Malden, 2009

ISBN 9780745631943 (hb) 9780745631950 (pb)

RRP $£ 55.00$ (hb) $£ 17.99$ (pb)

In her 2002 text, Young People and New Media: Childhood and the Changing Media Environment, Sonia Livingstone described the feeling that a defining moment in media audience research had arrived. Livingstone wrote that the development of an increasingly individualised and privatised media environment had transformed the practices that encompass media engagement, calling for new strategies to understand this engagement. Leisure, social interaction and citizenship, according to Livingstone, are experiences that occur, increasingly, within a media environment. New media, it seems, has the capacity to transform all areas of society. This capacity echoes throughout her 2009 text, Children and the Internet: Great Expectations, Challenging Realities. Livingstone identifies her guiding principle as an endeavour to understand the gap between the optimism that suggests that the internet has the capacity to allow children to be transformed by a media culture that invites their 
interaction, and the findings of empirical research, which suggest that these interactions involve challenges, risks and unfulfilled potential. (ix)

Throughout her text, Livingstone contextualises the relationship between children and the internet in a number of ways. The first two chapters offer an overview of how the internet affects the way that childhood is conceptualised. Later chapters develop the analytic narrative by focusing upon particular interconnected but distinct themes. The third chapter focuses upon how education is undergoing a transformation that is driven by developments in information and communication technologies. The following chapter examines how these technologies provide opportunities for the development of identity through online social interaction and leisure. The fifth chapter argues that the internet is an increasingly popular forum for civic participation, and that it may be an essential ingredient in facilitating the development of politically active citizens. The sixth chapter balances these opportunities against the various kinds of risk that are generated through online interaction. The last two chapters define media literacy and demonstrate how media literacy helps to manage risk and ensure that the potential benefits of online interaction come to fruition.

Childhood is undergoing a radical transformation in ways that are both complex and multifaceted. While the perception of childhood as a contingent category has been a popular thesis since the 1960s, Livingstone contributes to this through demonstrating that late modernity poses specific challenges to the relations between children and adults. 'Children's life circumstances are pressured, constrained [and] stratified' (60) and parents seem ill equipped to assist in these struggles. She argues that:

contemporary families must negotiate a rapidly changing society without the traditional resources of established relations between the generations, with parents neither benefiting from the experience of their own childhood nor having the moral right to impose rules and sanctions without democratic consultation. (7)

Information and communication technologies, subject to a range of intense developments in the past fifteen years, risk alienating parents from their children. Rather than exercising a prohibitionist attitude to media consumption, parents need to accept that the home is a 'node in a broader network that connects children's lives 
to a range of opportunities to learn, communicate, know [and] participate' (61) in the media environment. Accordingly, parents should endeavour to understand how new media technologies function in the lives of their children, rather than attempting to control access.

The capacity to access the internet is an issue that features prominently in debates about the welfare of children. In the popular imagination, the internet is conceptualised as an environment that, at its best, functions as a repository of knowledge that provides an educational benefit, and allows the user to develop skills that facilitate social and civic engagement. Accordingly, a lack of access to the internet is justifiably equated with exclusion. However, Livingstone's research suggests that access to the internet is not synonymous with possessing the skills to use the technology effectively. The gap between the potential of online interaction and the actual way in which young people use the internet is revealed through examining patterns of internet usage. Livingstone argues:

a fairly conservative pattern of use primarily defined by pre-existing interests and preferences, notwithstanding the huge diversity of possible activities and contents. These familiar use practices tend to be mass media-related (particularly through preferences for certain television programmes, popular music groups or football teams) or strongly branded-with entertainment, game playing and educational uses for young children easily managed through the provision of 'walled gardens'.

Accordingly, it appears that the 'digital natives' are as easily corralled as the 'digital immigrants', frequently opting for an online experience that promises relative familiarity and security, rather than exploring areas of the internet that elicit fear in the public imagination. However, as argued by Livingstone, the distinction between digital natives and digital immigrants is one that neglects to afford young people the guidance that they are usually afforded. Parents may believe (and often rightly so) that their children possess more skills for online interaction than they do. However, this fallacy confuses familiarity with acumen, and obscures the different skill levels that children possess, and subsequently the extent to which they are at risk. 
The risks children and young people face, online, manifest in various ways. Livingstone divides these into four broad categories: 'commercial, aggressive, sexual and values-related motivations'. (159) These risks are three fold, they can involve the child as recipient, participant and actor. For example, the sexual risks presented to children can be conceptualised in the following way. The first instance involves accidental exposure to pornographic or sexual content. The second instance involves situations where children interact with, or are groomed by, strangers. The third instance involves circumstances where children produce and distribute pornographic content of their own volition. However, maintaining a clear distinction between these roles can be difficult. For example, a child may be acting as a participant in a particular online interaction that leads to unwelcome exposure to pornography. A more cautious approach to interaction, which relies upon agency, may have avoided this exposure. Similarly, a child is transformed from a participant to an actor when they create and upload visual pornography. However, it seems that they remain a participant if they interact in a way that is similarly lascivious, but lacks the production and circulation of images. This distinction seems to revolve around the materiality of evidence more than a clear difference in the agency of the user. These distinctions can seem a little unclear, but Livingstone should be commended for her willingness to move beyond a simple dichotomy that characterises adults as perpetrators and children as victims.

Children are also susceptible to a more subtle, but nonetheless pertinent, risk that is connected to online interaction. The inability to sufficiently manage an online identity leaves children vulnerable. As Livingstone reports, online risks may arise from:

limited internet literacy combined with confusing or poorly designed site settings, leaving them unclear regarding their control over who can see what about them. (118)

Therefore, children are vulnerable to a number of risks that may be mitigated through the development of tools to enhance media literacy. Media literacy is described as a set of skills that allow individuals to interpret and communicate forms of knowledge. It is a practice where someone is empowered to critique rather than simply affirm forms of knowledge, to express agency in an immersed environment rather than simply acting as beneficiary of sets of skills and rules. The 
internet is a media environment that therefore has the capacity to function as more than simply a reinforcement of traditional pedagogy. Rather, it has the capacity to facilitate a radical shift in the way in which knowledge is accumulated, developed and distributed. While the best way to encourage media literacy is yet to be fully developed, the research that Livingstone has conducted, and draws upon throughout this text, suggests a way forward.

The empirical research that informs Livingstone's argument is sizable, drawing upon a number of studies conducted throughout the twenty-first century. The UK Children Go Online project was an empirical study conducted by Livingstone and Magdalena Bober. ${ }^{1}$ Involving surveys and focus groups with more than fifteen hundred children, aged nine to nineteen, and more than nine hundred parents, this project is a credible snapshot of the middle of the first decade of the twenty-first century. EU Kids Online is an ongoing research network, involving research teams across twenty-one countries and twenty-five thousand research participants. Livingstone and Leslie Haddon of the London School of Economics coordinated this project, which completed its first phase between 2006 and 2009.2 This attention to empiricism facilitates a child-centred approach to media culture. It listens to the experiences children have, and to how they make sense of them, in order to determine how adults can develop technology and policy that will enhance their interaction. This is a significant challenge for adults because the internet is a fluid network subject to developments that are driven by technology and the market. However, the failure to construct an environment to advance media literacy would be tantamount to abandoning efforts to help children acquire literacy. When media literacy is not acquired, it is not just that an opportunity to participate in the new media environment is lost. Rather, people face exclusion from important social and civic processes, and risk being victimised and manipulated by other parties who possess the requisite skills.

The text is quite accessible, as each chapter has a clearly defined focus. The chapters are thematically distinct and work in harmony to form a cogent argument. However, the use of sub-headings is a little inconsistent. Chapters three, four, five, and seven each close with a section marked 'Conclusions'. It's not clear why this device is not used consistently throughout the text. Despite this, the argument remains quite lucid, both within and across chapters. Furthermore, the text includes 
an appendix that offers details of research projects, directed by Livingstone, conducted between 1995 and 2007. The inclusion of this material emphasises that this publication is intended primarily for readers who are not necessarily familiar with the research projects with which Livingstone and her colleagues are involved, but wish to receive a succinct summary of the empirical bases for the claims made throughout the text. More than a summary, however, this text provides a clear argument that culminates in a series of practical recommendations that involve the responsibilities of parents, governments and the market.

This publication is not a theoretical departure from the concept of media audiences that Livingstone developed in her 2002 work Young People and New Media. But this is not to suggest that this text merely reiterates the goal, methodology or findings of this earlier publication. Rather, they complement one another as they both mark periods where Livingstone has taken the opportunity to reflect upon the implications that arise from current research in this area of media audience studies. Considering that her bibliography contains more than fifty entries that describe her as single or co-author, Livingstone's expertise in this area is evident. Although the background research that frames her hypothesis should be familiar to anyone with an interest in children as media audience members, the core research presented in this text is more than sufficient to warrant the publication of this text. While Livingstone avoids a celebratory approach to the changing media environment, readers have cause to celebrate the publication of a text that offers an accessible evaluation of the field. Livingstone's research may alleviate concerns, finding that the current situation is not as grim as some people fear. However, her argument suggests that adults need to take greater responsibility to ensure that children are offered a safe environment and, more importantly, that they are bestowed the skills that will allow them to navigate and create, and make their own choices about how they will shape their media environment.

Adam Stapleton is a PhD candidate in the school of Communication Arts at the University of Western Sydney. His dissertation examines typologies of child pornography. He is a member of Onscenity, located at http://www.onscenity.org. 
Additional research interests include horror films, low-brow art and other forms of loathsome entertainment.

\section{-NOTES}

${ }^{1}$ S. Livingstone and M. Bober, UK Children Go Online: Final Report of Key Project Findings, LSE Research Online, London, 2005, <http://eprints.lse.ac.uk/399/1/UKCGO_Final_report.pdf>, accessed 1 April 2011.

2 S. Livingstone and L. Haddon, EU Kids Online: Final Report, EU Kids Online, LSE, London, 2009, <http://www2.lse.ac.uk/media@lse/research/EUKidsOnline/EU\%20Kids\%20I\%20(2006-

9)/EU\%20Kids\%200nline\%20I\%20Reports/EUKidsOnlineFinalReport.pdf>, accessed 1 April 2011. 